\title{
On the Triangular Decomposition of Cauchy Matrices
}

\section{By Choong Yun Cho}

1. Introduction. Recently Schechter [4], and (apparently independently) Trench and Scheinok [5] have shown that the elements of the inverse of a Cauchy matrix may be written down in simple closed form.

In this short note we demonstrate that the triangular decomposition of the Cauchy matrix may also be written out explicitly, and furthermore that the same is true of the inverse of these triangular factors.

The inverses of the triangular factors are deduced from new determinantal expressions for the elements of the triangular factors of a general matrix.

2. Preliminary Results. It is convenient to use the following notation for the general square matrix $A$ of order $k$ :

$$
A=\left(a_{1} b_{2} c_{3} \cdots v_{k-1} w_{k}\right)=\left[\begin{array}{ccccc}
a_{1} & b_{1} & c_{1} & \ldots & w_{1} \\
a_{2} & b_{2} & c_{2} & \cdots & w_{2} \\
& & \cdot & & \\
& & \cdot & & \\
& & & & \\
a_{k} & b_{k} & c_{k} & \ldots & w_{k}
\end{array}\right] .
$$

We denote the determinant of this matrix by $\left|a_{1} b_{2} c_{3} \cdots w_{k}\right|$. Thus

$$
\left|b_{1} d_{3} h_{4}\right|=\operatorname{det}\left[\begin{array}{lll}
b_{1} & d_{1} & h_{1} \\
b_{3} & d_{3} & h_{3} \\
b_{4} & d_{4} & h_{4}
\end{array}\right] .
$$

It is known (Turnbull [6, p. 369]) that for the usual triangular decomposition $A=L U$, the elements of $L$ and $U$ can be expressed in terms of determinants involving the elements of $A$ as follows (it is assumed that the decomposition is possible and $L$ has been chosen to have units in the principal diagonal):

$$
L=\left[\begin{array}{ccccc}
1 & & & & \\
\frac{\left|a_{2}\right|}{\left|a_{1}\right|} & 1 & & & \\
\frac{\left|a_{3}\right|}{\left|a_{1}\right|} & \frac{\left|a_{1} b_{3}\right|}{\left|a_{1} b_{2}\right|} & 1 & & \\
& \ldots & & & \\
\frac{\left|a_{m}\right|}{\left|a_{1}\right|} & \frac{\left|a_{1} b_{m}\right|}{\left|a_{1} b_{2}\right|} & \frac{\left|a_{1} b_{2} c_{m}\right|}{\left|a_{1} b_{2} c_{3}\right|} & \ldots & \\
\frac{\left|a_{k}\right|}{\left|a_{1}\right|} & \frac{\left|a_{1} b_{k}\right|}{\left|a_{1} b_{2}\right|} & \frac{\left|a_{1} b_{2} c_{k}\right|}{\left|a_{1} b_{2} c_{3}\right|} & \frac{\left|a_{1} b_{2} c_{3} d_{k}\right|}{\left|a_{1} b_{2} c_{3} d_{4}\right|} & \ldots
\end{array}\right]
$$

Received September 5, 1967. Revised January 16, 1968. 
and

(2) $U=$

$$
\left[\begin{array}{l}
\left|a_{1}\right| \quad\left|b_{1}\right| \\
\frac{\left|a_{1} b_{2}\right|}{\left|a_{1}\right|} \\
\end{array}\right.
$$

$$
\left.\begin{array}{cccc}
\left|c_{1}\right| & \left|d_{1}\right| & \cdots & \left|w_{1}\right| \\
\frac{\left|a_{1} c_{2}\right|}{\left|a_{1}\right|} & \frac{\left|a_{1} d_{2}\right|}{\left|a_{1}\right|} & \cdots & \frac{\left|a_{1} w_{2}\right|}{\left|a_{1}\right|} \\
\frac{\left|a_{1} b_{2} c_{3}\right|}{\left|a_{1} b_{2}\right|} & \frac{\left|a_{1} b_{2} d_{3}\right|}{\left|a_{1} b_{2}\right|} & \cdots & \frac{\left|a_{1} b_{2} w_{3}\right|}{\left|a_{1} b_{2}\right|} \\
\cdot \\
\cdot \\
\\
& & \frac{\left|a_{1} b_{2} \cdots v_{k-1} w_{k}\right|}{\left|a_{1} b_{2} \cdots v_{k-1}\right|}
\end{array}\right]
$$

It does not seem to have been noticed that $L^{-1}$ and $U^{-1}$ can similarly be expressed explicitly as follows:

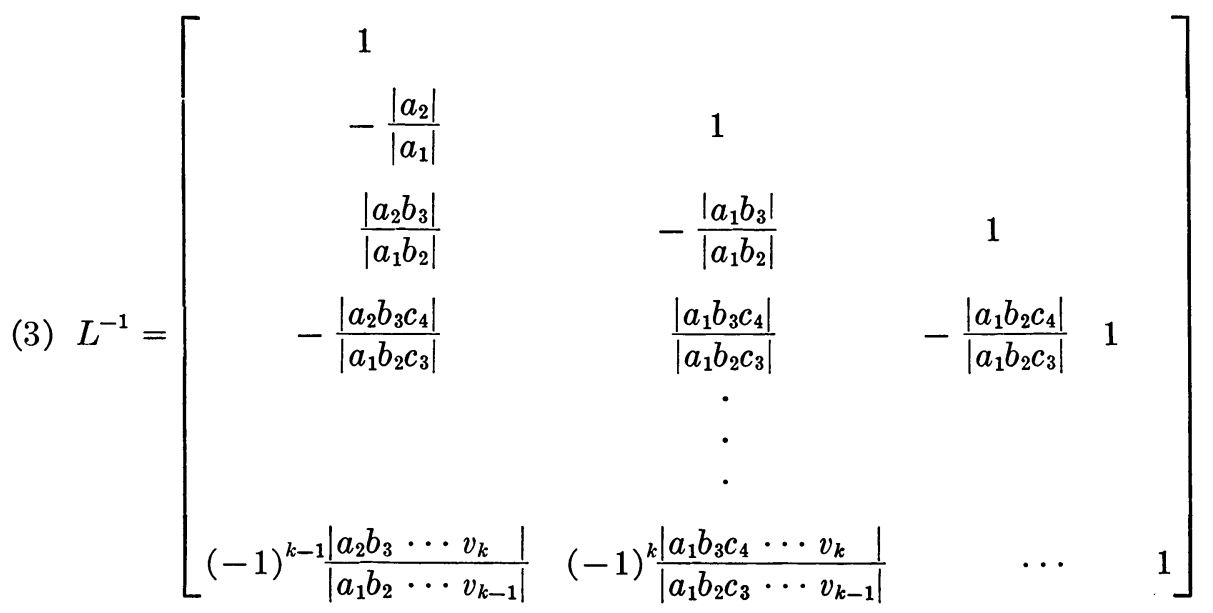

and

(4) $U^{-1}=$

$$
\left[\begin{array}{rrr}
\frac{|1|}{\left|a_{1}\right|}-\frac{\left|b_{1}\right|}{\left|a_{1} b_{2}\right|}-\frac{\left|b_{1} c_{2}\right|}{\left|a_{1} b_{2} c_{3}\right|}-\frac{\left|b_{1} c_{2} d_{3}\right|}{\left|a_{1} b_{2} c_{3} d_{4}\right|} \cdots(-1)^{k-1} \frac{\left|b_{1} c_{2} d_{3} \cdots w_{k-1}\right|}{\left|a_{1} b_{2} c_{3} \cdots w_{k}\right|} \\
\frac{\left|a_{1}\right|}{\left|a_{1} b_{2}\right|}-\frac{\left|a_{1} c_{2}\right|}{\left|a_{1} b_{2} c_{3}\right|} & \frac{\left|a_{1} c_{2} d_{3}\right|}{\left|a_{1} b_{2} c_{3} d_{4}\right|} \cdots(-1)^{k} \frac{\left|a_{1} c_{2} d_{3} \cdots w_{k-1}\right|}{\left|a_{1} b_{2} c_{3} \cdots w_{k}\right|} \\
\frac{\left|a_{1} b_{2}\right|}{\left|a_{1} b_{2} c_{3}\right|}-\frac{\left|a_{1} b_{2} d_{3}\right|}{\left|a_{1} b_{2} c_{3} d_{4}\right|} \cdots(-1)^{k+1} \frac{\left|a_{1} b_{2} d_{3} \cdots w_{k-1}\right|}{\left|a_{1} b_{2} c_{3} \cdots w_{k}\right|} & \cdot \\
\cdot \\
\cdot \\
\frac{\left|a_{1} b_{2} c_{3} \cdots v_{k-1}\right|}{\left|a_{1} b_{2} c_{3} \cdots w_{k}\right|}
\end{array}\right]
$$


These results can be shown as follows (the author is indebted to the referee for this simple and elegant proof):

Since $L^{-1} A=U$, the requirement that the product $L^{-1} A$ be upper triangular imposes $(m-1)$ conditions on the $(m-1)$ elements of $L^{-1}$ in the $m$ th row of $L^{-1} A$, i.e.,

$$
\left(L^{-1} A\right)_{m s}=0 \quad(m>s)
$$

or

$$
\sum_{r=1}^{m-1}\left(L^{-1}\right)_{m r} A_{r s}=-A_{m s}, \quad s=1,2, \cdots, m-1 .
$$

This is a system of $(m-1)$ equations for the unknowns $\left(L^{-1}\right)_{m r}$, $r=1,2, \cdots, m-1$, and formula (3) for $L^{-1}$ is merely Cramer's rule.

Similarly, we have

$$
\left(A U^{-1}\right)_{\Theta m}=0 \quad(m>s)
$$

or

$$
\sum_{r=1}^{m-1} A_{s r}\left(U^{-1}\right)_{r m}=-A_{s m}\left(U^{-1}\right)_{m m}, \quad s=1,2, \cdots, m-1 .
$$

But (see [2, p. 11])

$$
\left(U^{-1}\right)_{m m}=\frac{\left|a_{1} b_{2} \cdots g_{m-1}\right|}{\left|a_{1} b_{2} \cdots g_{m-1} h_{m}\right|},
$$

and by the same argument expression (4) for $U^{-1}$ is obtained from Cramer's rule.

In some problems one wishes to decompose a matrix into a product of the form $U L$ rather than in the more usual form $L U$. If now we begin with $A=U L$, where $A$ is the general square matrix of order $k, A=\left(a_{1} b_{2} c_{3} \cdots v_{k-1} w_{k}\right)$, then we obtain by the same method

$$
U=\left[\begin{array}{ccccc}
\frac{\left|a_{1} b_{2} c_{3} d_{4} \cdots w_{k}\right|}{\left|b_{2} c_{3} d_{4} \cdots w_{k}\right|} & \frac{\left|b_{1} c_{3} d_{4} \cdots w_{k}\right|}{\left|c_{3} d_{4} \cdots w_{k}\right|} & \frac{\left|c_{1} d_{4} \cdots w_{k}\right|}{\left|d_{4} \cdots w_{k}\right|} \cdots \frac{\left|v_{1} w_{k}\right|}{\left|w_{k}\right|} & \left|w_{1}\right| \\
& \frac{\left|b_{2} c_{3} d_{4} \cdots w_{k}\right|}{\left|c_{3} d_{4} \cdots w_{k}\right|} & \frac{\left|c_{2} d_{4} \cdots w_{k}\right|}{\left|d_{4} \cdots w_{k}\right|} \cdots \frac{\left|v_{2} w_{k}\right|}{\left|w_{k}\right|} & \left|w_{2}\right| \\
& \frac{\left|c_{3} d_{4} \cdots w_{k}\right|}{\left|d_{4} \cdots w_{k}\right|} \cdots & \frac{\left|v_{3} w_{k}\right|}{\left|w_{k}\right|} & \left|w_{3}\right| \\
& & \cdot & \\
& & & \\
& & & \\
& & & \left|v_{k-1} w_{k}\right| & \left|w_{k-1}\right| \\
& & & \left|w_{k}\right|
\end{array}\right]
$$

and 


$$
\text { (6) } \quad L=\left[\begin{array}{cccc}
1 & & & \\
\frac{\left|a_{2} c_{3} d_{4} e_{5} \cdots w_{k}\right|}{\left|b_{2} c_{3} d_{4} e_{5} \cdots w_{k}\right|} & 1 & & \\
\frac{\left|a_{3} d_{4} e_{5} \cdots w_{k}\right|}{\left|c_{3} d_{4} e_{5} \cdots w_{k}\right|} & \frac{\left|b_{3} d_{4} e_{5} \cdots w_{k}\right|}{\left|c_{3} d_{4} e_{5} \cdots w_{k}\right|} & 1 & \\
\frac{\left|a_{4} e_{5} \cdots w_{k}\right|}{\left|d_{4} e_{5} \cdots w_{k}\right|} & \frac{\left|b_{4} e_{5} \cdots w_{k}\right|}{\left|d_{4} e_{5} \cdots w_{k}\right|} & \frac{\left|c_{4} e_{5} \cdots w_{k}\right|}{\left|d_{4} e_{5} \cdots w_{k}\right|} & 1 \\
\frac{\left|a_{k}\right|}{\left|w_{k}\right|} & \frac{\left|b_{k}\right|}{\left|w_{k}\right|} & \frac{\left|c_{k}\right|}{\left|w_{k}\right|} & \cdots
\end{array}\right]
$$

Their inverses are

(7) $U^{-1}=$

$$
\left[\begin{array}{r}
\frac{\left|b_{2} c_{3} d_{4} \cdots w_{k}\right|}{\left|a_{1} b_{2} c_{3} d_{4} \cdots w_{k}\right|}-\frac{\left|b_{1} c_{3} d_{4} \cdots w_{k}\right|}{\left|a_{1} b_{2} c_{3} d_{4} \cdots w_{k}\right|} \\
\frac{\left|c_{3} d_{4} \cdots w_{k}\right|}{\left|b_{2} c_{3} d_{4} \cdots w_{k}\right|}
\end{array}\right.
$$$$
\left.\begin{array}{rr}
\frac{\left|b_{1} c_{2} d_{4} \cdots w_{k}\right|}{\left|a_{1} b_{2} c_{3} d_{4} \cdots w_{k}\right|} \cdots(-1)^{k-1} \frac{\left|b_{1} c_{2} d_{3} \cdots w_{k-1}\right|}{\left|a_{1} b_{2} c_{3} d_{4} \cdots w_{k}\right|} \\
-\frac{\left|c_{2} d_{4} \cdots w_{k}\right|}{\left|b_{2} c_{3} d_{4} \cdots w_{k}\right|} \cdots & (-1)^{k} \frac{\left|c_{2} d_{3} \cdots w_{k-1}\right|}{\left|b_{2} c_{3} d_{4} \cdots w_{k}\right|} \\
\frac{\left|d_{4} \cdots w_{k}\right|}{\left|c_{3} d_{4} \cdots w_{k}\right|} \cdots & (-1)^{k+1} \frac{\left|d_{3} \cdots w_{k-1}\right|}{\left|c_{3} d_{4} \cdots w_{k}\right|} \\
\cdot & \cdot \\
\cdot & \frac{|1|}{\left|w_{k}\right|}
\end{array}\right]
$$

and

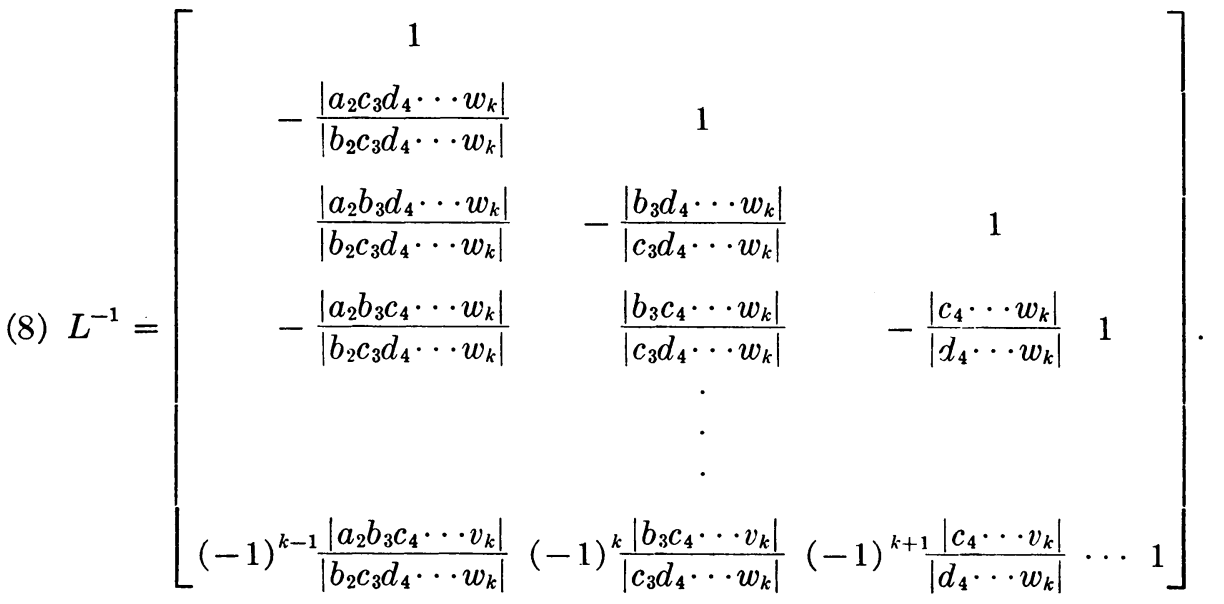

These expressions, Eq. (1) to Eq. (8) inclusively, constitute a complete set of 
the formulas for the triangular decomposition of a general square matrix, expressed in terms of determinants.

3. Main Results. We denote the Cauchy matrix of order $k$

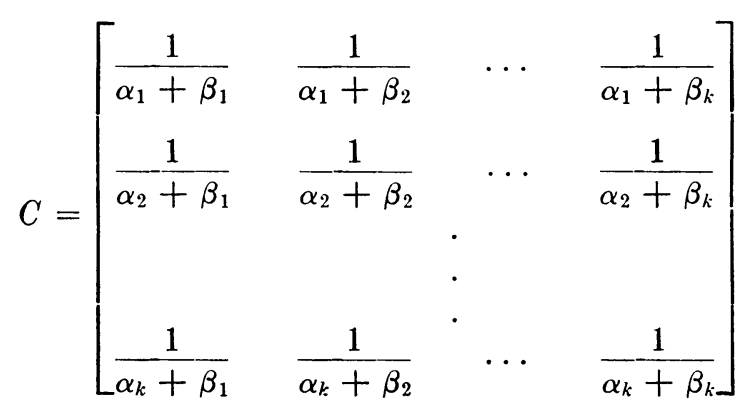

by $\left(\left(1 /\left(\alpha_{m}+\beta_{n}\right) ; m, n=1,2, \cdots, k\right)\right)$.

Theorem 1. Given the Cauchy matrix of order $k, \quad C=\left(\left(1 /\left(\alpha_{m}+\beta_{n}\right) ; m\right.\right.$, $n=1,2, \cdots, k)$ ), where $\alpha_{1}, \alpha_{2}, \cdots, \alpha_{k} ;-\beta_{1},-\beta_{2}, \cdots,-\beta_{k}$, are pairwise distinct and $C^{\prime}=L U$. Then the triangular factors are

$$
\begin{aligned}
& L_{m, n}=\frac{\left(\alpha_{n}+\beta_{n}\right)}{\left(\alpha_{m}+\beta_{n}\right)} \cdot \prod_{s=1}^{n-1} \frac{\left(\alpha_{n}+\beta_{s}\right)\left(\alpha_{m}-\alpha_{s}\right)}{\left(\alpha_{m}+\beta_{s}\right)\left(\alpha_{n}-\alpha_{s}\right)}, \\
& U_{m, n}=\frac{1}{\left(\alpha_{m}+\beta_{n}\right)} \cdot \prod_{s=1}^{m-1} \frac{\left(\alpha_{m}-\alpha_{s}\right)\left(\beta_{n}-\beta_{s}\right)}{\left(\alpha_{s}+\beta_{n}\right)\left(\alpha_{m}+\beta_{s}\right)} .
\end{aligned}
$$

Proof. We merely have to substitute $\left(\alpha_{m}+\beta_{n}\right)^{-1}$ in Eq. (1) and observe that the $m$ th row of $L$ has elements formed by the quotients of two determinants. Each numerator is obtained by replacing the last row of the matrix whose determinant is in the denominator with the corresponding elements of the $m$ th row of the original given matrix. Note that $m$ is always larger than the order of the determinant under consideration.

Using the formula for the determinant of Cauchy form (see, e.g., Pólya and Szegö [3, p. 98]), a simple arithmetic manipulation yields formula (9). Formula (10) can be derived in an analogous fashion.

In a very similar way we prove

TheOREM 2. The elements of the matrix inverses of $L$ and $U$, where $C=L U$, are given by

$$
\begin{aligned}
& \left(L^{-1}\right)_{m, n}=\prod_{t=1 ; t \neq n}^{m} \frac{1}{\left(\alpha_{n}-\alpha_{t}\right)} \cdot \prod_{s=1}^{m-1} \frac{\left(\alpha_{m}-\alpha_{s}\right)\left(\alpha_{n}+\beta_{s}\right)}{\left(\alpha_{m}+\beta_{s}\right)} \quad(m \geqq n), \\
& \left(U^{-1}\right)_{m, n}=\frac{\left(\alpha_{n}+\beta_{n}\right)}{\prod_{t=1 ; t \neq m}^{n}\left(\beta_{m}-\beta_{t}\right)} \cdot \prod_{s=1}^{n-1} \frac{\left(\alpha_{s}+\beta_{m}\right)\left(\alpha_{n}+\beta_{s}\right)}{\left(\alpha_{n}-\alpha_{s}\right)} \quad(m \leqq n) .
\end{aligned}
$$

Theorem 3. Given the Cauchy matrix $C$ of order $k$ as defined in Theorem 1, let $C=U L$, then

$$
U_{m, n}=\frac{1}{\left(\alpha_{m}+\beta_{n}\right)} \cdot \prod_{s=n+1}^{k} \frac{\left(\alpha_{m}-\alpha_{s}\right)\left(\beta_{n}-\beta_{s}\right)}{\left(\alpha_{s}+\beta_{n}\right)\left(\alpha_{m}+\beta_{s}\right)},
$$




$$
\begin{aligned}
L_{m, n} & =\frac{\left(\alpha_{m}+\beta_{m}\right)}{\left(\alpha_{m}+\beta_{n}\right)} \cdot \prod_{s=m+1}^{k} \frac{\left(\alpha_{s}+\beta_{m}\right)\left(\beta_{n}-\beta_{s}\right)}{\left(\alpha_{s}+\beta_{n}\right)\left(\beta_{m}-\beta_{s}\right)}, \\
\left(U^{-1}\right)_{m, n} & =\frac{\left(\alpha_{m}+\beta_{m}\right)}{\prod_{t=m ; t \neq n}^{k}\left(\alpha_{n}-\alpha_{t}\right)} \cdot \prod_{s=m+1}^{k} \frac{\left(\alpha_{n}+\beta_{s}\right)\left(\alpha_{s}+\beta_{m}\right)}{\left(\beta_{m}-\beta_{s}\right)} \quad(m \leqq n), \\
\left(L^{-1}\right)_{m, n} & =\prod_{t=n ; t \neq m}^{k} \frac{1}{\left(\beta_{m}-\beta_{t}\right)} \cdot \prod_{s=n+1}^{k} \frac{\left(\alpha_{s}+\beta_{m}\right)\left(\beta_{n}-\beta_{s}\right)}{\left(\alpha_{s}+\beta_{n}\right)} \quad(m \geqq n) .
\end{aligned}
$$

The proof of this theorem can be shown by the same sort of elementary manipulations used to establish Theorems 1 and 2 .

(Remark. All the empty products in above expressions are assumed to be unity.)

4. Hankel and Toeplitz Matrices. In two special cases it turns out that the Cauchy matrix reduces to one of Toeplitz form: These occur when

$$
T_{1}=((1 /(m-n+x) ; m, n=1,2, \cdots, k))
$$

where $x$ is a constant, and

$$
T_{2}=\left(\left(1 /\left(p-q^{m-n+x}\right) ; m, n=1,2, \cdots, k\right)\right)
$$

where $p, q$, and $x$ are constants.

In the first case we obtain readily $L, U, L^{-1}$, and $U^{-1}$ by putting $\alpha_{m}=m$ and $\beta_{n}=x-n$. The inverse becomes

$$
\left(T_{1}^{-1}\right)_{m, n}=\frac{\prod_{t=1}^{k}(x+t-m)(x-t+n)}{(n-m+x) \cdot \prod_{r=1 ; r \neq m}^{k}(m-r) \cdot \prod_{s=1 ; s \neq n}^{k}(s-n)} .
$$

In the second case we note that

$$
T_{2}=\left(\left(1 /\left(p q^{n}-q^{m+x}\right) ; m, n=1,2, \cdots, k\right)\right) \cdot D,
$$

where $D$ is a diagonal matrix with $d_{n n}=q^{n}, n=1,2, \cdots, k$.

The first factor of $T_{2}$ now has the Cauchy form, i.e., $\alpha_{m}=-q^{m+x}$ and $\beta_{n}=p q^{n}$, and the triangular factors are readily expressed. The inverse of $T_{2}$ is

$$
\left(T_{2}^{-1}\right)_{m, n}=\frac{\prod_{t=1}^{k}\left(p q^{m}-q^{x+t}\right)\left(p q^{t}-q^{x+n}\right)}{y \cdot \prod_{r=1 ; r \neq m}^{k}\left(q^{r}-q^{m}\right) \cdot \prod_{s=1 ; s \neq n}^{k}\left(q^{n}-q^{s}\right)}
$$

where $y=p^{k} q^{k x-x+2 m}-p^{k-1} q^{k x+m+n}$.

The Hankel forms corresponding to the above Toeplitz matrices $T_{1}$ and $T_{2}$ are

$$
H_{1}=((1 /(m+n+x) ; m, n=1,2, \cdots, k))
$$

and

$$
H_{2}=\left(\left(1 /\left(p-q^{m+n+x}\right) ; m, n=1,2, \cdots, k\right)\right),
$$

respectively.

The triangular factors of $H_{1}$ and $H_{2}$, and their inverses are immediately obtained by choosing $\alpha_{m}$ and $\beta_{n}$ in the theorems. In particular, the inverse of $H_{2}$ is 


$$
\left(H_{2}^{-1}\right)_{m, n}=\frac{\prod_{t=1}^{k}\left(p q^{-m}-q^{x+t}\right)\left(p q^{-t}-q^{x+n}\right)}{y \cdot \prod_{r=1 ; r \neq m}^{k}\left(q^{-r}-q^{-m}\right) \cdot \prod_{s=1 ; s \neq n}^{k}\left(q^{n}-q^{s}\right)}
$$

where

$$
y=p^{k} q^{k x-x-2 m}-p^{k-1} q^{k x-m+n} .
$$

5. Acknowledgement. The author is indebted to Professor B. Noble, University of Wisconsin, and Professor P. Wynn, Mathematics Research Center, University of Wisconsin, for their guidance and encouragement throughout the project.

Maggs Research Center

U. S. Army Arsenal

Watervliet, New York 12189

1. A. C. Aitrken, Determinants and Matrices, Interscience, New York, 1964.

2. A. S. Housenolder, The Theory of Matrices in Numerical Analysis, Blaisdell, New York, 1964. MR $30 \# 5475$.

3. G. PóLYA \& G. Szegö, Aufgaben und Lehrsätze aus der Analysis, Vol. II, Springer-Verlag, Berlin, 1964. MR $30 \# 1219 \mathrm{~b}$.

4. S. ScheCHTER, "On the inversion of certain matrices," Math. Comp., v. 13, 1959, pp. 73-77. MR $21 \# 4534$.

5. W. F. Trench \& P. A. Scheinok, "On the inversion of a Hilbert type matrix," SIAM Rev., v. 8, 1966, pp. 57-61. MR $33 \# 8092$.

6. H. W. TuRnBull, The Theory of Determinants, Matrices, and Invariants, Dover, New York, 1960. MR 24 \#A123. 